\title{
Validation of the SNACOR clinical scoring system after transarterial chemoembolisation in patients with hepatocellular carcinoma
}

Aline Mähringer-Kunz ${ }^{1}$, Arndt Weinmannn ${ }^{2,3}$, Irene Schmidtmann ${ }^{4}$, Sandra Koch³, Sebastian Schotten', Daniel Pinto dos Santos ${ }^{5}$, Michael Bernhard Pitton ${ }^{1}$, Christoph Dueber ${ }^{1}$, Peter Robert Galle ${ }^{2}$

and Roman Kloeckner ${ }^{1 *}$ (D)

\begin{abstract}
Background: Transarterial chemoembolisation is the standard of care for intermediate stage (BCLC B) hepatocellular carcinoma, but it is challenging to decide when to repeat or stop treatment. Here we performed the first external validation of the SNACOR (tumour Size and Number, baseline Alpha-fetoprotein, Child-Pugh and Objective radiological Response) risk prediction model.

Methods: A total of 1030 patients with hepatocellular carcinoma underwent transarterial chemoembolisation at our tertiary referral centre from January 2000 to December 2016. We determined the following variables that were needed to calculate the SNACOR at baseline: tumour size and number, alpha-fetoprotein level, Child-Pugh class, and objective radiological response after the first transarterial chemoembolisation. Overall survival, timedependent area under receiver-operating characteristic curves, Harrell's C-index, and the integrated Brier score were calculated to assess predictive ability. Finally, multivariate analysis was performed to identify independent predictors of survival.

Results: The study included 268 patients. Low, intermediate, and high SNACOR scores predicted a median survival of 31.5, 19.9, and 9.2 months, respectively. The areas under the receiver-operating characteristic curve for overall survival were $0.641,0.633$, and 0.609 at 1, 3, and 6 years, respectively. Harrell's C-index was 0.59 , and the integrated Brier Score was 0.175 . Independent predictors of survival included tumour size $(P<0.001)$, baseline alpha-fetoprotein level $(P<0.001)$ and Child-Pugh class $(P<0.004)$. Objective radiological response $(P=0.821)$ and tumour number $(P=0.127)$ were not additional independent predictors of survival.
\end{abstract}

Conclusions: The SNACOR risk prediction model can be used to identify patients with a dismal prognosis after the first transarterial chemoembolisation who are unlikely to benefit from further transarterial chemoembolisation. However, Harrell's C-index showed only moderate performance. Accordingly, this risk prediction model can only serve as one of several components used to make the decision about whether to repeat treatment.

Keywords: Hepatocellular carcinoma, Transarterial chemoembolisation, SNACOR

\footnotetext{
*Correspondence: roman.kloeckner@gmail.com;

roman.kloeckner@unimedizin-mainz.de

'Department of Diagnostic and Interventional Radiology, Johannes

Gutenberg-University Medical Center Mainz, Langenbeckst. 1, 55131 Mainz,

Germany

Full list of author information is available at the end of the article
}

(c) The Author(s). 2018 Open Access This article is distributed under the terms of the Creative Commons Attribution 4.0 International License (http://creativecommons.org/licenses/by/4.0/), which permits unrestricted use, distribution, and

reproduction in any medium, provided you give appropriate credit to the original author(s) and the source, provide a link to the Creative Commons license, and indicate if changes were made. The Creative Commons Public Domain Dedication waiver (http://creativecommons.org/publicdomain/zero/1.0/) applies to the data made available in this article, unless otherwise stated. 


\section{Background}

Hepatocellular carcinoma (HCC) is one of the most common cancers worldwide and the second most common cause of cancer-related deaths $[1,2]$. According to the Barcelona Clinic Liver Cancer (BCLC) classification, transarterial chemoembolisation (TACE) is the recommended treatment for intermediate-stage HCC (BCLCB) [3]. However, the BCLC-B subgroup is quite heterogeneous, and not all patients benefit equally from TACE [4]. The question of when to stop TACE and possibly change to systemic treatment or even to best supportive care remains a challenge. In recent years, several scoring systems have been developed to support decision making after the first TACE, including the ART score (Assessment for Retreatment with TACE) and the ABCR score (Alpha-fetoprotein, BCLC, Child-Pugh, and Response) $[5,6]$. However, none of these scoring systems are currently used in clinical practice.

To provide decision support regarding the issue of TACE retreatment, Kim et al. recently introduced the SNACOR (tumour Size, tumour Number, baseline Alpha-fetoprotein level, Child-Pugh class, and Objective radiological Response) clinical scoring system [7]. This system uses baseline liver function, baseline tumour parameters, and tumour response after the first TACE to evaluate the suitability of retreatment. However, the use of such clinical scoring systems in clinical routine has been controversial, and further external validation has been recommended $[8,9]$. A few studies have been conducted to validate the ART score [10-14] and the ABCR score [13], but, to the best of our knowledge, no attempt has been made to validate the SNACOR score. Therefore, the purpose of this study was to perform the first external validation of the SNACOR score.

\section{Methods}

\section{Patients}

The study was approved by the institutional review board (IRB) for the retrospective analysis of clinical data. Patient records and clinical information were deidentified prior to analysis. Primary data collection was carried out using specially developed clinical registry software for the characterisation of patients with HCC [15].

The inclusion and exclusion criteria were the same as in the original SNACOR publication. The study included treatment-naive patients who received TACE as first-line therapy and who had HCC diagnosed by histological or radiological evaluation according to the American Association for the Study of Liver Diseases (AASLD) or the European Association for the Study of the Liver (EASL) guidelines [7, 16, 17]. The study excluded patients with an inadequate target lesion (infiltrative pattern, non-arterial enhancement, or largest lesion < $1 \mathrm{~cm}$ ); patients with an additional primary malignancy in another organ or with extrahepatic lesions; Child-Pugh class $\mathrm{C}$ patients; and patients with uncontrolled functional or metabolic disease [7].

As recommended by the authors of the original SNACOR publication, who only included patients who underwent conventional TACE, patients in this study received conventional, Lipiodol-based TACE (cTACE), or TACE using drug-eluting beads (DEB-TACE) [7]. Treatment was performed in a standardised manner that is extensively described elsewhere [18, 19].

\section{Imaging and tumour response}

Each patient underwent contrast-enhanced computed tomography (CT) or magnetic resonance imaging (MRI) prior to the first TACE treatment. Six weeks after the first TACE treatment, restaging with $\mathrm{CT}$ or MRI was performed prior to the second TACE. This examination was the basis for the radiological assessment of the tumour response, which was evaluated by applying the unidimensional EASL criteria [20]. The objective tumour response was defined as a partial response (PR) before the second TACE treatment. Stable disease (SD) and progressive disease (PD) were assessed as a lack of radiological response.

\section{Calculation of the SNACOR score}

The SNACOR score consists of the summed scores of the following variables: tumour size $(<5 \mathrm{~cm}, 0$ points; $\geq 5 \mathrm{~cm}, 1$ point), tumour number $(<4,0$ points; $\geq 4,2$ points), baseline alpha-fetoprotein level $(<400 \mathrm{ng} / \mathrm{ml}, 0$ points; $\geq 400 \mathrm{ng} / \mathrm{ml}, 3$ points), Child-Pugh class (A, 0 points; $\mathrm{B}, 1$ point), and the objective radiological response $(C R+P R, 0$ points; $S D+P D, 3$ points $)$. Hence, the SNACOR score ranges from 0 to 10 points. According to the original SNACOR paper, three risk groups can be differentiated using the SNACOR score: 0-2 points, low risk; 3-6 points, intermediate risk; and 7-10 points, high risk [7].

\section{Statistical analysis}

Overall survival (OS) was defined as the period from the day before the first TACE until death or last follow-up. Kaplan-Meier survival curves were drawn using R 3.4.2 (A Language and Environment for Statistical Computing, R Foundation for Statistical Computing, https:// www.R-project.org; accessed 2017). Survival between strata was compared using the log-rank test. Kernel probability densities were obtained using the $\mathrm{R}$ package survPresmooth, which calculates presmoothed probability density estimates for censored data [21]. Cumulative/dynamic receiver operating characteristic (ROC) curves were obtained using the $\mathrm{R}$ package timeROC. Areas under the curve (AUROCs) were derived at specified time points for comparison with those in the original SNACOR paper. 
R 3.4.2 and SAS 9.4 were used for descriptive statistics and to perform multivariate analyses of all variables used in the SNACOR system in order to identify independent predictors of survival and to calculate hazard ratios (HRs) with corresponding $95 \%$ confidence intervals (CIs). As this analysis was intended to be exploratory, the $P$-values should be interpreted in a descriptive manner.

Validation was performed using Harrell's C-index, and prediction error curves were based on the Brier score $[22,23]$. Both Harrell's C-index and AUROC can range from 0 to 1 , where 0.5 indicates no predictive ability and 1 indicates perfect predictive ability. A value below 0.5 indicates "anti-prediction". The Brier score at time $t$ is the mean squared difference between the observed outcome ( 1 for event and 0 otherwise) and the predicted outcome probability at time $t$. The integrated Brier score (IBS) over the interval $[0 \mathrm{~m}, 72 \mathrm{~m}]$ was calculated as a summary measure of prediction error.

\section{Results}

\section{Patient recruitment}

A total of 1030 patients with HCC underwent TACE between January 2000 and December 2016 at our tertiary referral centre, and 762 patients were excluded for the reasons shown in the CONSORT flowchart (Fig. 1). Thus, the SNACOR score was calculated for 268 patients.

\section{Baseline patient characteristics and treatment}

In our cohort, the mean patient age prior to the first TACE was 66.5 years (median, 66.9 years; range, 36. $1-87.3$ years; SD \pm 9.4$)$. A total of $227(84.7 \%)$ patients were men, and $41(15.3 \%)$ were women. The main aetiology of HCC was alcohol abuse. Table 1 shows the baseline patient characteristics of our cohort and those of the original SNACOR cohort. CTACE was performed in 190 patients, and DEB-TACE was performed in 78 patients. Overall, the mean number of TACE sessions was 5.6 (median, 5; min, 1; max, 21).

\section{SNACOR score}

All variables that were needed to calculate the SNACOR score (both at baseline and prior to the second TACE) were determined (Table 1). Of the 268 patients, 94 (35.1\%) were in the low-risk SNACOR score group (score 0-2), 144 patients (53.7\%) were in the intermediate-risk group (score 3-6), and 30 patients (11.2\%) were in the high-risk group (score 7-10). The median OS was 31.5 months (95\% CI 23.1-46.0) in the low-risk group, 19.9 months (95\% CI 17.1-26.2) in the intermediate-risk group, and 9.2 months in the high-risk group (95\% CI 6.2-21.7). The Kaplan-Meier survival curves are shown in Fig. 2. Table 2 compares the survival

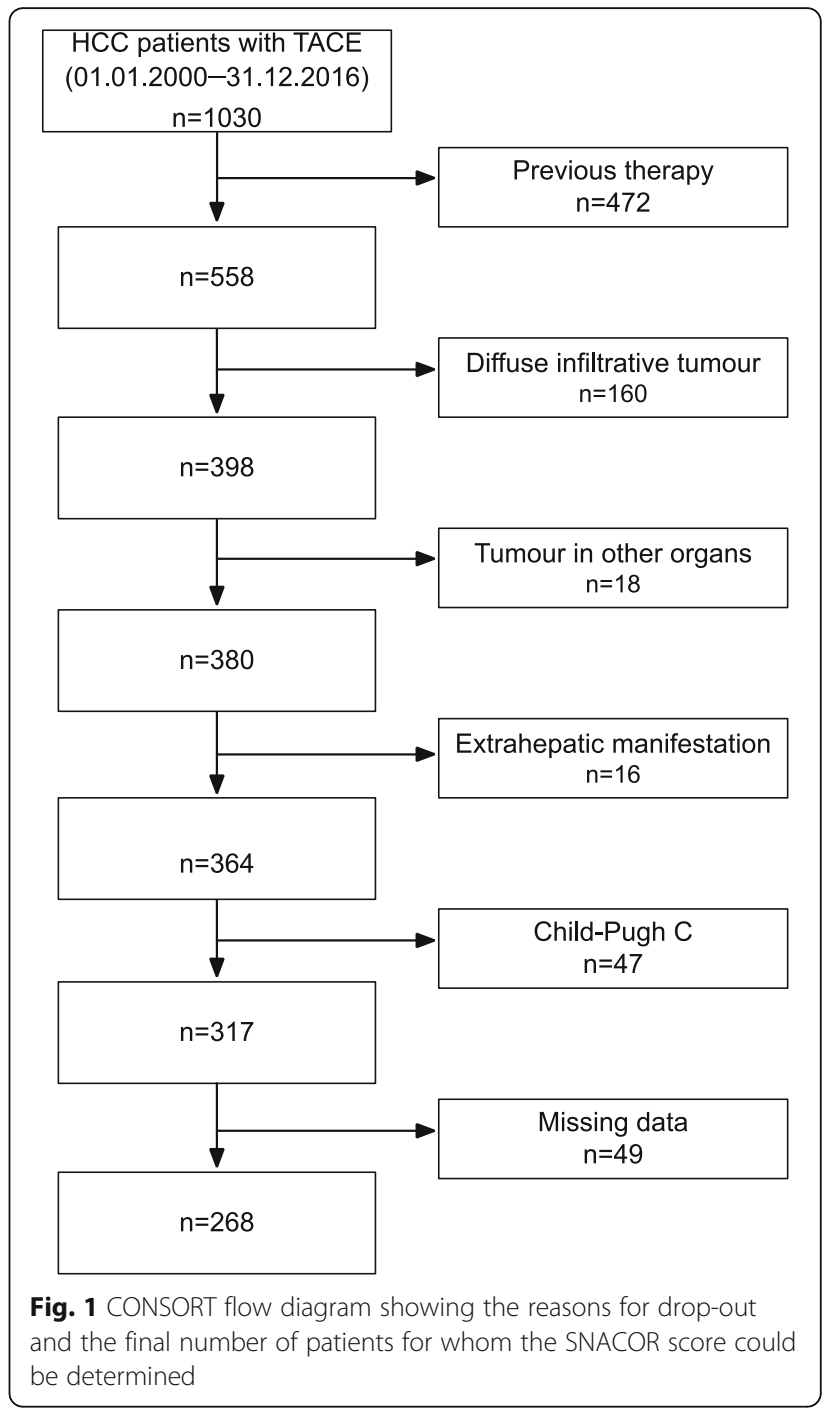

rates in our study with those in the original SNACOR study [7].

The AUROC for overall survival was 0.641 at 1 year, 0 . 633 at 3 years, and 0.609 at 6 years. Harrell's C-index was 0.59 . The prediction error curves are shown in Fig. 3. The IBS for the first 6 years was 0.175 . In comparison, the IBS was 0.184 using the Kaplan-Meier estimates for the unstratified sample. The probability density estimates (Fig. 4) show a high degree of overlap.

The Cox regression analysis used tumour size, tumour number, baseline alpha-fetoprotein level, the Child-Pugh class, and objective radiological response as covariates (Table 3). Only tumour size, baseline alpha-fetoprotein level, and the Child-Pugh class had significant prognostic value $(\mathrm{HR}=2.51, P<0.001 ; \mathrm{HR}=1.76, P<0.001 ; \mathrm{HR}=1$. $56, P=0.004)$. Objective radiological response $(\mathrm{HR}=0$. 97, $P=0.821)$ and tumour number $(\mathrm{HR}=1.28, P=0$. 127) were not additional independent predictors of survival. 
Table 1 Baseline characteristics of patients with hepatocellular carcinoma in this study and in the original SNACOR study [7]

\begin{tabular}{|c|c|c|c|c|c|}
\hline & & \multicolumn{2}{|l|}{ This study } & \multicolumn{2}{|c|}{ Original SNACOR study } \\
\hline & & $n=268$ & $\%$ & $n=340$ & $\%$ \\
\hline \multicolumn{6}{|l|}{ Prior to first TACE } \\
\hline \multirow[t]{2}{*}{ Age, y } & Mean \pm SD & $66.5 \pm 9.4$ & & 58 & \\
\hline & Range & $36.1-87.3$ & & $51-65$ & \\
\hline \multirow[t]{2}{*}{ Sex } & Male & 227 & 84.7 & 274 & 80.6 \\
\hline & Female & 41 & 15.3 & 66 & 19.4 \\
\hline \multirow[t]{5}{*}{ Aetiology ${ }^{a}$} & Alcohol & 134 & 50.0 & & \\
\hline & Hepatitis B virus & 24 & 9.0 & 242 & 71.2 \\
\hline & Hepatitis C virus & 77 & 28.7 & 44 & 12.9 \\
\hline & Other $^{\mathrm{b}}$ & 42 & 15.7 & 54 & 15.9 \\
\hline & No underlying liver disease & 9 & 3.3 & 0 & 0 \\
\hline \multirow[t]{2}{*}{ Child Pugh stage } & A & 184 & 68.7 & 288 & 84.7 \\
\hline & B & 84 & 31.3 & 52 & 15.3 \\
\hline \multirow[t]{2}{*}{ Tumour size, mm } & Mean \pm SD & $52 \pm 35$ & & 53 & \\
\hline & Range & $10-215$ & & $27-88$ & \\
\hline \multirow[t]{5}{*}{ Number of nodes } & 1 & 78 & 29.1 & 127 & 37.4 \\
\hline & 2 & 80 & 29.9 & 74 & 21.8 \\
\hline & 3 & 44 & 16.4 & 31 & 9.1 \\
\hline & 4 & 36 & 13.4 & 31 & 9.1 \\
\hline & $\geq 5$ & 30 & 11.2 & 77 & 22.6 \\
\hline \multirow[t]{2}{*}{ Alpha-fetoprotein, ng/ml } & Median & 30.5 & & 120.0 & \\
\hline & Range & $0.5-920,910$ & & $17.1-1430.0$ & \\
\hline
\end{tabular}

${ }^{a}$ the sum of aetiologies is $>100 \%$ because patients could have two or more aetiologies

buther" comprises: nonalcoholic steatohepatitis $(n=17 ; 6.3 \%)$, cryptogenic liver cirrhosis $(n=14 ; 5.2 \%)$, hemochromatosis $(n=11 ; 4.1 \%)$

\section{Discussion}

In this study, the SNACOR score was able to differentiate between low-, intermediate-, and high-risk patients, who respectively showed a median OS of 31.5 months, 19.9 months, and 9.2 months. However, the original SNACOR publication reported respective median OS values of 49.8 months, 30.7 months, and 12.4 months for these groups. Hence, the discriminative ability of the SNACOR score between the three risk groups with respect to OS was inferior in our study compared to the original one. We observed considerable overlap in the survival time distribution. Accordingly, the Harrell's Cindex was 0.59 and the IBS was 0.175. AUROCs for overall survival were 0.641 at 1 year, 0.633 at 3 years, and 0.609 at 6 years; in the original SNACOR study, the comparable AUROC values were 0.756, 0.754, and 0.742, respectively. In summary, SNACOR does not perform well enough to be used alone to make clear-cut clinical decisions.

In the multivariate analysis, and in contrast to the original SNACOR study, we were only able to confirm the predictive value of tumour size, baseline alphafetoprotein level, and Child-Pugh class. Thus, two of the five parameters for calculating the SNACOR score were

Fig. 2 Kaplan-Meier survival curves according to SNACOR score category $(n=268)$ and log-rank test $p$-value 
Table 2 Comparison of the survival rates of patients with hepatocellular carcinoma in this study versus the survival rates of patients in the original SNACOR study

\begin{tabular}{lllll}
\hline SNACOR, 3 subgroups & Low risk, 0-2 points & Intermediate risk, 3-6 points & High risk, 7-10 points & P-value \\
\hline This study: median OS $(95 \% \mathrm{Cl}), \mathrm{m}$ & $31.5(23.1-46.0)$ & $19.9(17.1-26.2)$ & $9.2(6.2-21.7)$ & $<0.001$ \\
Original SNACOR study: median OS (95\% Cl), $\mathrm{m}$ & $49.8(34.3-65.3)$ & $30.7(25.8-35.6)$ & $12.4(5.9-18.9)$ & $<0.001$ \\
\hline
\end{tabular}

not predictive in our analysis, which may at least in part be due to the moderate sample size. The objective radiological response and tumour number at baseline failed to show a significant impact on survival. Notably, tumour size and tumour number reflect a patient's tumour burden, and tumour size correlates with a higher risk of vascular invasion and distant metastasis [24, 25]. As tumour size is a known independent risk factor of survival $[26,27]$, it is part of several risk prediction models that have been published in recent years. We confirmed that tumour size is an independent predictor of survival. However, as noted above, tumour number was not an additional independent predictor of survival in our analysis. Whether or not tumour number is a significant prognostic factor is unclear in the literature; some series found it to have predictive value [27-30], while others did not $[5,26]$. The fact that tumour number was not an independent predictor of survival in our study collective might be attributable to the moderate size of the final patient group of 268 patients. However, this validation group was considerably bigger than the validation cohort in the original SNACOR publication, which comprised 145 patients. Furthermore, it might be

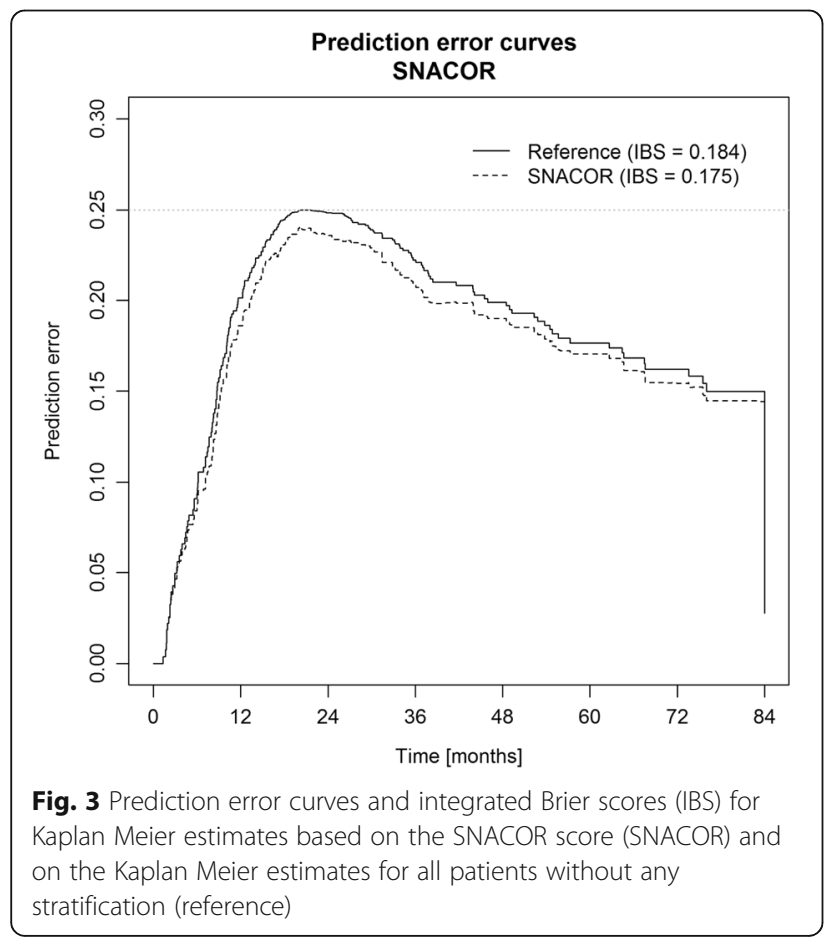

explained at least in part by the phenomenon of collinearity; we observed some positive correlation between tumour size and tumour number (Spearman $r=0.165$ ). Alpha-fetoprotein level (AFP) was an independent predictor of survival in our analysis, which is in accordance with the majority of publications [27-29, 31], since AFP may be a surrogate marker for tumour burden and tumour aggressiveness $[32,33]$. Therefore, AFP is part of several prediction scores $[6,26,30]$. The Child-Pugh score describes liver function and has shown significant prognostic value in several studies [28, 34-36]. Objective radiological response was not an additional independent predictor of survival in our analysis. Although it was not predictive in several other studies as well $[10,37]$, most authors regard objective radiological response as an important predictor $[5,6,31,38]$. The fact that objective radiological response was not an independent predictor in our study might also be attributable to the moderate sample size and the phenomenon of collinearity, at least in part. We observed a weak negative correlation between tumour size and the objective radiological response (Spearman $r=-0.172$ ). One important reason why the SNACOR score did not show the same predictive power in our study as in the original publication might be the so-called "overfitting" effect. This has been described as "a phenomenon occurring when a model

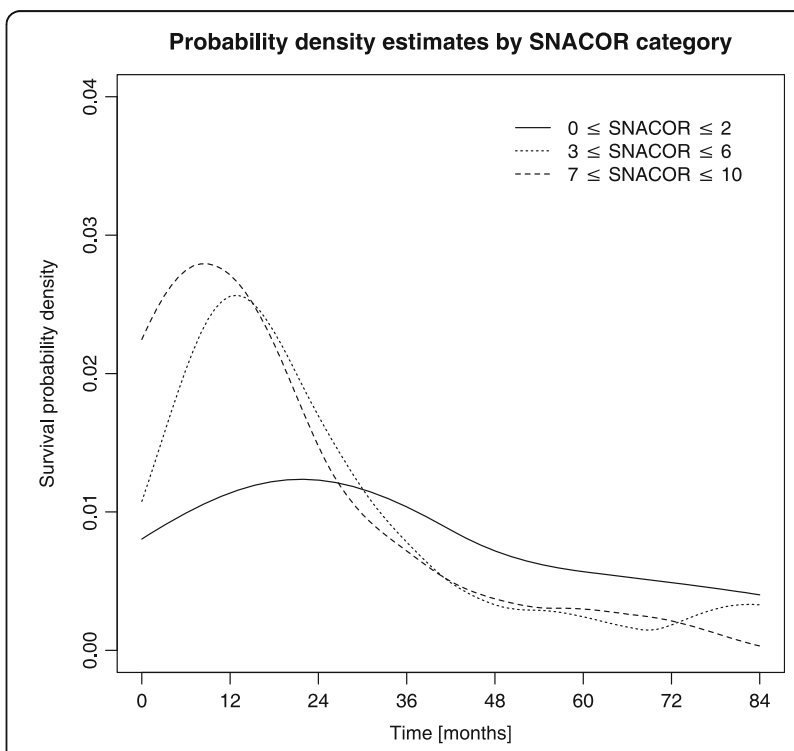

Fig. 4 Presmoothed kernel estimates of the survival probability density according to SNACOR category $(n=268)$ 
Table 3 Proportional hazards model to identify independent predictors of survival and to compare hepatocellular carcinoma patient data in this study to the data of patients in the original SNACOR study [7]

\begin{tabular}{|c|c|c|c|c|}
\hline & SNACOR parameters & & Hazard ratio $(95 \% \mathrm{Cl})$ & $P$-value \\
\hline \multirow[t]{5}{*}{ This study } & Tumour size & $\geq 5 \mathrm{~cm}$ vs. $<5 \mathrm{~cm}$ & $2.51(1.88-3.36)$ & $<0.001$ \\
\hline & Tumour number & $\geq 4$ vs. $<4$ & $1.28(0.93-1.75)$ & 0.127 \\
\hline & Baseline AFP level & $\geq 400 \mathrm{ng} / \mathrm{ml}$ vs. $<400 \mathrm{ng} / \mathrm{ml}$ & $1.76(1.28-2.43)$ & $<0.001$ \\
\hline & Child-Pugh class & A vs. B & $1.56(1.16-2.12)$ & 0.004 \\
\hline & Objective radiological response & $C R+P R$ vs. $S D+P D$ & $0.97(0.73-1.28)$ & 0.821 \\
\hline \multirow[t]{5}{*}{ Original SNACOR study } & Tumour size & $\geq 5 \mathrm{~cm}$ vs. $<5 \mathrm{~cm}$ & $1.29(0.95-0.17)$ & 0.100 \\
\hline & Tumour number & $\geq 4$ vs. $<4$ & $1.68(1.24-2.28)$ & 0.001 \\
\hline & Baseline AFP level & $\geq 400 \mathrm{ng} / \mathrm{ml} \mathrm{vs} .<400 \mathrm{ng} / \mathrm{ml}$ & $2.09(1.55-2.82)$ & $<0.001$ \\
\hline & Child-Pugh class & A vs. B & $1.44(0.96-2.14)$ & 0.074 \\
\hline & Objective radiological response & $C R+P R$ vs. $S D+P D$ & $2.24(1.65-3.03)$ & $<0.001$ \\
\hline
\end{tabular}

maximizes its performance on some set of data but its predictive performance is not confirmed elsewhere due to random fluctuations of patients' characteristics in different clinical and demographical backgrounds [8]". Our patients differed significantly from the patients in the original SNACOR study in terms of tumour number, Child-Pugh class, and aetiology [7]. For example, alcoholic cirrhosis was the main reason for hepatocellular carcinoma in our study, whereas in the study by Kim et al., 71.2\% of patients had hepatitis-B-related hepatocellular carcinoma, and $12.9 \%$ of patients had hepatitis-Crelated hepatocellular carcinoma [7].

Our analysis has several limitations. The most important ones are that our validation was conducted in a retrospective manner and that the final sample size $(n=$ 268) was only moderate. Ideally, prospective validation would be performed with a sufficiently large patient cohort using a multicentre approach. As recommended by the authors of the original SNACOR publication, which only included patients who underwent cTACE, in this study TACE was performed as cTACE or using DEBTACE. Differences in TACE techniques might influence the applicability of the SNACOR system. cTACE and DEB-TACE have been compared multiple times in the last decade, but these comparisons have never shown a significant influence on survival $[18,39,40]$. Indeed, we drew the same conclusion when we analysed our own data [41]. Patients who underwent liver transplantation or surgery after TACE were excluded in the present analysis in order to ensure comparability with the original SNACOR data. However, from a statistical point of view, such patients should not be excluded; rather, they should be censored at the time of treatment change in order to eliminate immortal time bias.

\section{Conclusions}

Even though the SNACOR system showed some ability to discriminate between patients with a favourable outcome after TACE versus patients with an impaired prognosis, SNACOR alone was not sufficient to reliably distinguish different prognostic groups. Therefore, SNACOR alone is not sufficient to support clear-cut clinical decision making, and further efforts are needed to determine appropriate criteria for making valid clinical predictions. Other approaches, such as machine learning, could be helpful for making future clinical predictions with increased validity.

\section{Abbreviations}

AASLD: American association for the study of liver diseases; ABCR: Alphafetoprotein, BCLC, child-pugh, and response; AFP: Alpha-fetoprotein; ART: Assessment for retreatment with TACE; AUROC: Areas under receiver operating characteristic; BCLC: Barcelona clinic liver cancer classification; Cl: Confidence interval; CT: Computed tomography; CTACE: Conventional transarterial chemoembolisation,; DEB-TACE: Drug-eluting bead transarterial chemoembolisation; EASL: European association for the study of the liver; HCC: Hepatocellular carcinoma; HR: Hazard ratio; IBS: Integrated brier score; IRB: Institutional review board; MRI: Magnetic resonance imaging; OS: Overall survival; PD: Progressive disease; PR: Partial response; ROC: Receiver operating characteristic; SD: Stable disease; SNACOR: Tumour size, tumour number, baseline alpha-fetoprotein level, child-pugh class, and objective radiological response; TACE: Transarterial chemoembolisation

\section{Acknowledgments}

We wish to thank Ms. Katherine Taylor for her contribution to the language editing of this manuscript. We further acknowledge financial support from the German Research Foundation (DFG) and Johannes Gutenberg -

University through the funding program for Open Access Publishing.

\section{Funding}

No funding was obtained for this study.

\section{Availability of data and materials}

The data that support the findings of this study are included within the article. The primary data are stored in an internal clinical registry software specially developed for the clinical characterisation of patients with HCC to ensure participant confidentiality. The data are available upon request from the corresponding author.

\section{Authors' contributions}

AMK, AW, IS, SS, MBP, CD, PRG, DPDS, and RK devised the study, assisted in data collection, participated in the interpretation of the data, and helped draft the manuscript. AMK and RK carried out the data collection. AW, SK, DPDS, and IS supported the data collection efforts. IS and SK created all of 
the figures and participated in the interpretation of data. IS performed the statistical analysis. All authors read and approved the final manuscript.

\section{Ethics approval and consent to participate}

Institutional review board approval was waived by the responsible Ethics Committee of the Medical Association of Rhineland Palatinate, Mainz, Germany, given the retrospective study design and analysis of clinical data. Additional examinations were not performed. Patient records and information were anonymized and de-identified prior to analysis.

\section{Competing interests}

PRG has received grants and personal fees from Bayer, personal fees from Bristol-Myers Squibb, personal fees from MSD Sharp \& Dohme, personal fees from Lilly, personal fees from Sillajen, personal fees from SIRTEX, and personal fees from AstraZeneca. IS has received grants from Merck Serono. AW has received speaker fees and travel grants from Bayer. RK has received speaker fees from SIRTEX and personal fees from Guerbet and Bristol-Myers Squibb. None of these companies supported this study and none of the authors reports a conflict of interest.

\section{Publisher's Note}

Springer Nature remains neutral with regard to jurisdictional claims in published maps and institutional affiliations.

\section{Author details}

'Department of Diagnostic and Interventional Radiology, Johannes Gutenberg-University Medical Center Mainz, Langenbeckst. 1, 55131 Mainz, Germany. ${ }^{2}$ Department of Internal Medicine, Johannes Gutenberg-University Medical Center Mainz, Mainz, Germany. ${ }^{3}$ Clinical Registry Unit (CRU), Johannes Gutenberg-University Medical Center Mainz, Mainz, Germany. ${ }^{4}$ Institute of Medical Biostatistics, Epidemiology and Informatics, Johannes Gutenberg-University Mainz, Mainz, Germany. ${ }^{5}$ Department of Radiology, University Hospital Cologne, Cologne, Germany.

Received: 20 December 2017 Accepted: 18 April 2018 Published online: 27 April 2018

\section{References}

1. Torre LA, Bray F, Siegel RL, Ferlay J, Lortet-Tieulent J, Jemal A. Global cancer statistics, 2012. CA Cancer J Clin. 2015;65:87-108.

2. Ferlay J, Soerjomataram I, Dikshit R, Eser S, Mathers C, Rebelo M, et al. Cancer incidence and mortality worldwide: sources, methods and major patterns in GLOBOCAN 2012. Int J Cancer. 2015;136:359-86.

3. Llovet JM, Bru C, Bruix J. Prognosis of hepatocellular carcinoma: the BCLC staging classification. Semin Liver Dis. 1999;19:329-38.

4. Bolondi L, Burroughs A, Dufour JF, Galle PR, Mazzaferro V, Piscaglia F, et al. Heterogeneity of patients with intermediate (BCLC B) hepatocellular carcinoma: proposal for a subclassification to facilitate treatment decisions. Semin Liver Dis. 2012;32:348-59.

5. Sieghart W, Hucke F, Pinter M, Graziadei I, Vogel W, Muller C, et al. The ART of decision making: retreatment with transarterial chemoembolization in patients with hepatocellular carcinoma. Hepatology. 2013;57:2261-73.

6. Adhoute X, Penaranda G, Naude S, Raoul JL, Perrier H, Bayle O, et al. Retreatment with TACE: the ABCR SCORE, an aid to the decisionmaking process. J Hepatol. 2015;62:855-62.

7. Kim BK, Shim JH, Kim SU, Park JY, Kim do Y, Ahn SH, et al. Risk prediction for patients with hepatocellular carcinoma undergoing chemoembolization: development of a prediction model. Liver Int. 2016;36:92-9.

8. Facciorusso A, Bhoori S, Sposito C, Mazzaferro V. Repeated transarterial chemoembolization: an overfitting effort? J Hepatol. 2015;62:1440-2.

9. Fatourou EM, Tsochatzis EAART. Science in using transarterial chemoembolization for retreating patients with hepatocellular carcinoma. Hepatobiliary Surg Nutr. 2014;3:415-8.

10. Terzi E, Terenzi L, Venerandi L, Croci L, Renzulli M, Mosconi C, et al. The ART score is not effective to select patients for transarterial chemoembolization retreatment in an Italian series. Dig Dis. 2014;32(6):711.

11. Arizumi T, Ueshima K, Iwanishi M, Minami T, Chishina H, Kono M, et al. Evaluation of ART scores for repeated Transarterial chemoembolization in Japanese patients with hepatocellular carcinoma. Oncology. 2015;89(Suppl 2):4-10.
12. Yin W, Ye Q, Wang F, Liang J, Xu B, Zhang X, et al. ART score and hepatocellular carcinoma: an appraisal of its applicability. Clin Res Hepatol Gastroenterol. 2016;40:705-14.

13. Kloeckner R, Pitton MB, Dueber C, Schmidtmann I, Galle PR, Koch S, et al. Validation of clinical scoring systems ART and ABCR after Transarterial chemoembolization of hepatocellular carcinoma. J Vasc Interv Radiol. 2017;28:94-102

14. Ha Y, Lee JB, Shim JH, Kim KM, Lim YS, Yoon HK, et al. Validation and reappraisal of the assessment for retreatment with transarterial chemoembolization score for unresectable non-metastatic hepatocellular carcinoma in a hepatitis b virus-endemic region. Eur Radiol. 2016;26:3510-8.

15. Weinmann A, Koch S, Niederle IM, Schulze-Bergkamen H, Konig J, Hoppe-Lotichius $M$, et al. Trends in epidemiology, treatment, and survival of hepatocellular carcinoma patients between 1998 and 2009: an analysis of 1066 cases of a German HCC registry. J Clin Gastroenterol. 2014;48:279-89.

16. European Association For The study of the $L$, European organisation for $R$, treatment of C. EASL-EORTC clinical practice guidelines: management of hepatocellular carcinoma. J Hepatol. 2012;56:908-43.

17. Bruix J, Sherman M, American Association for the Study of liver D. Management of hepatocellular carcinoma: an update. Hepatology. 2011;53:1020-2.

18. Lammer J, Malagari K, Vogl T, Pilleul F, Denys A, Watkinson A, et al. Prospective randomized study of doxorubicin-eluting-bead embolization in the treatment of hepatocellular carcinoma: results of the PRECISION V study. Cardiovasc Intervent Radiol. 2010;33:41-52.

19. Lencioni R, de Baere T, Burrel M, Caridi JG, Lammer J, Malagari K, et al. Transcatheter treatment of hepatocellular carcinoma with doxorubicinloaded DC bead (DEBDOX): technical recommendations. Cardiovasc Intervent Radiol. 2012;35(5):980.

20. Lencioni R, Llovet JM. Modified RECIST (mRECIST) assessment for hepatocellular carcinoma. Semin Liver Dis. 2010;30:52-60.

21. Lopez de Ullibarri I, Jacome MA. survPresmooth: an R package for Presmoothed estimation in survival analysis. J Stat Softw. 2013;54:1-26.

22. Gerds TA, Schumacher M. Efron-type measures of prediction error for survival analysis. Biometrics. 2007;63:1283-7.

23. Mogensen UB, Ishwaran $H$, Gerds TA. Evaluating random forests for survival analysis using prediction error curves. J Stat Softw. 2012;50:1-23.

24. Fukutomi S, Nomura $Y$, Nakashima $O$, Yano H, Tanaka H, Akagi Y, et al. Evaluation of hepatocellular carcinoma spread via the portal system by 3dimensional mapping. HPB (Oxford). 2017;19:1119-25.

25. Yokoo T, Patel AD, Lev-Cohain N, Singal AG, Yopp AC, Pedrosa I. Extrahepatic metastasis risk of hepatocellular carcinoma based on alpha-fetoprotein and tumor staging parameters at cross-sectional imaging. Cancer Manag Res. 2017;9:503-11.

26. Kadalayil L, Benini R, Pallan L, O'Beirne J, Marelli L, Yu D, et al. A simple prognostic scoring system for patients receiving transarterial embolisation for hepatocellular cancer. Ann Oncol. 2013;24:2565-70.

27. Cappelli A, Cucchetti A, Cabibbo G, Mosconi C, Maida M, Attardo S, et al. Refining prognosis after trans-arterial chemo-embolization for hepatocellular carcinoma. Liver Int. 2016;36:729-36.

28. Ha Y, Han S, Shim JH, Ko GY, Yoon HK, Sung KB, et al. Nomograms for predicting outcomes after chemoembolization in patients with nonmetastatic hepatocellular carcinoma. J Vasc Interv Radiol. 2015;26:1093-101.

29. Xu L, Peng ZW, Chen MS, Shi M, Zhang YJ, Guo RP, et al. Prognostic nomogram for patients with unresectable hepatocellular carcinoma after transcatheter arterial chemoembolization. J Hepatol. 2015;63:122-30.

30. Park Y, Kim SU, Kim BK, Park JY, Kim do Y, Ahn SH, et al. Addition of tumor multiplicity improves the prognostic performance of the hepatoma arterialembolization prognostic score. Liver Int. 2016;36:100-7.

31. Ichikawa T, Machida N, Sasaki H, Tenmoku A, Kaneko H, Negishi R, et al. Early prediction of the outcome using tumor markers and mRECIST in Unresectable hepatocellular carcinoma patients who underwent Transarterial chemoembolization. Oncology. 2016;91:317-30.

32. Okuda H, Nakanishi T, Takatsu K, Saito A, Hayashi N, Yamamoto M, et al. Comparison of clinicopathological features of patients with hepatocellular carcinoma seropositive for alpha-fetoprotein alone and those seropositive for des-gamma-carboxy prothrombin alone. J Gastroenterol Hepatol. 2001;16:1290-6.

33. Bai DS, Zhang C, Chen P, Jin SJ, Jiang GQ. The prognostic correlation of AFP level at diagnosis with pathological grade, progression, and survival of patients with hepatocellular carcinoma. Sci Rep. 2017;7:12870. 
34. Kim HC, Suk KT, Kim DJ, Yoon JH, Kim YS, Baik GH, et al. Transarterial chemoembolization in Barcelona clinic liver Cancer stage 0/a hepatocellular carcinoma. World J Gastroenterol 2014 21;20:745-754.

35. Ray CE Jr, Brown AC, Green TJ, Winston H, Curran C, Kreidler SM, et al. Survival outcomes in patients with advanced hepatocellular carcinoma treated with drug-eluting bead chemoembolization. AJR Am J Roentgenol. 2015;204:440-7.

36. Ogasawara S, Chiba T, Ooka Y, Kanogawa N, Motoyama T, Suzuki E, et al. A prognostic score for patients with intermediate-stage hepatocellular carcinoma treated with transarterial chemoembolization. PLoS One. 2015;10:e0125244.

37. Sawhney S, Montano-Loza AJ, Salat P, McCarthy M, Kneteman N, MezaJunco J, et al. Transarterial chemoembolization in patients with hepatocellular carcinoma: predictors of survival. Can J Gastroenterol. 2011;25:426-32.

38. Kim CJ, Kim HJ, Park JH, Park DI, Cho YK, Sohn Cl, et al. Radiologic response to transcatheter hepatic arterial chemoembolization and clinical outcomes in patients with hepatocellular carcinoma. Liver Int. 2014;34:305-12.

39. Sacco R, Bargellini I, Bertini M, Bozzi E, Romano A, Petruzzi P, et al. Conventional versus doxorubicin-eluting bead transarterial chemoembolization for hepatocellular carcinoma. J Vasc Interv Radiol. 2011;22:1545-52.

40. Golfieri R, Giampalma E, Renzulli M, Cioni R, Bargellini I, Bartolozzi C, et al. Randomised controlled trial of doxorubicin-eluting beads vs conventional chemoembolisation for hepatocellular carcinoma. Br J Cancer. 2014;111:255-64.

41. Kloeckner R, Weinmann A, Prinz F, Pinto dos Santos D, Ruckes C, Dueber C, et al. Conventional transarterial chemoembolization versus drug-eluting bead transarterial chemoembolization for the treatment of hepatocellular carcinoma. BMC Cancer. 2015;15:465.

Ready to submit your research? Choose BMC and benefit from:

- fast, convenient online submission

- thorough peer review by experienced researchers in your field

- rapid publication on acceptance

- support for research data, including large and complex data types

- gold Open Access which fosters wider collaboration and increased citations

- maximum visibility for your research: over $100 \mathrm{M}$ website views per year

At BMC, research is always in progress.

Learn more biomedcentral.com/submissions 